\title{
High resolution luminescence spectroscopy and thermoluminescence of different size $\mathrm{LaPO}_{4}: \mathrm{Eu}^{3+}$ nanoparticles
}

\author{
Tamara Gavrilovića $^{\mathrm{a}, \mathrm{b}, *}$, Katrīna Laganovska ${ }^{\mathrm{a}}$, Aleksejs Zolotarjovs ${ }^{\mathrm{a}}$, Krisjanis Smits ${ }^{\mathrm{a}}$, \\ Dragana J. Jovanović ${ }^{\mathrm{b}}$, Miroslav D. Dramićanin ${ }^{\mathrm{b}}$ \\ ${ }^{a}$ Institute of Solid State Physics, University of Latvia, 8 Kengaraga Street, Riga, LV-1063, Latvia \\ ${ }^{\mathrm{b}}$ Vinča Institute of Nuclear Sciences, University of Belgrade, P.O. Box 522, 11001, Belgrade, Serbia
}

\section{A R T I C L E I N F O}

\section{Keywords:}

$\mathrm{LaPO}_{4}: \mathrm{Eu}^{3+}$

High resolution spectroscopy

X-ray excitation

Thermo-stimulated luminescence

\begin{abstract}
A B S T R A C T
Nanoparticles $(5 \mathrm{~nm})$ and nanorods $(2 \mathrm{~nm} \times 15 \mathrm{~nm}$ and $4 \mathrm{~nm} \times 20 \mathrm{~nm})$ of monoclinic monazite $\mathrm{LaPO}_{4}: \mathrm{Eu}^{3+}$ were prepared by reverse micelle and co-precipitation techniques. Effects of the particle size and surface defects on the intensity of luminescence and the emission spectrum shapes were analyzed by high resolution spectroscopy under laser $(266 \mathrm{~nm})$ and X-rays excitation. All synthesized $\mathrm{LaPO}_{4}: \mathrm{Eu}^{3+}$ samples showed similar spectral features with characteristic $\mathrm{Eu}^{3+}$ ions emission bands: ${ }^{5} \mathrm{D}_{0} \rightarrow{ }^{7} \mathrm{~F}_{0}$ centered at $578.4 \mathrm{~nm}$, magnetic-dipole transition ${ }^{5} \mathrm{D}_{0} \rightarrow{ }^{7} \mathrm{~F}_{1}$ at 588-595 nm, electric-dipole transition ${ }^{5} \mathrm{D}_{0} \rightarrow{ }^{7} \mathrm{~F}_{2}$ at $611.5-620.5 \mathrm{~nm},{ }^{5} \mathrm{D}_{0} \rightarrow{ }^{7} \mathrm{~F}_{3}$ at $(648-652 \mathrm{~nm})$ and ${ }^{5} \mathrm{D}_{0} \rightarrow{ }^{7} \mathrm{~F}_{4}$ at $(684-702.5 \mathrm{~nm})$, with the most dominant electric-dipole ${ }^{5} \mathrm{D}_{0} \rightarrow{ }^{7} \mathrm{~F}_{2}$ transition. Additionally, the thermally stimulated luminescence was studied for the most dominant peak at $611.5 \mathrm{~nm}$. It was shown that the $\mathrm{Eu}^{3+}$ doping creates traps in all samples. Two prominent and well resolved glow peaks at $58.7 \mathrm{~K}$ and $172.3 \mathrm{~K}$ were detected for $5 \mathrm{~nm}$ nanoparticles, while low-intensity glow-peaks at $212.1 \mathrm{~K}$ and $212.2 \mathrm{~K}$ were observed in the X-rays irradiated nanorods. Displayed glows could be attributed to free and bound electrons and holes or to the recombination of electrons of ionized oxygen vacancies with photogenerated holes. To obtain information about the processes and specific defect type it is necessary to carry out additional analysis for all synthesized samples. The glow curves were analyzed and trap parameters were estimated and discussed throughout the paper.
\end{abstract}

\section{Introduction}

Nowadays, luminescent materials doped with lanthanide ions play an important role in everyday life due to their unique chemical, structural and physicochemical properties. They are characterized by high energy conversion efficiency, purity in spectral colors, strong emission, high thermal stability and conductivity, and could be applied in optical devices such as scintillators, solid-state lighting, lasers, cathode ray tubes (CRTs), electroluminescent, field emission and flat panel display devices, chemical and temperature sensors [1-5].

It is well-known, that the defects from the nanoparticles' surface affect luminescence properties of nanomaterials. The defect luminescence exhibits a strong dependence on the temperature and excitation wavelength, with some defect emissions observable only at low temperatures and for certain excitation wavelengths [6]. Many different species have been involved, including vacancies, holes, interstitial oxygen defects and electron traps or self-trapped excitons [7]. High surface to volume ratio of nanoparticles plays a major role in the concentration of ionized oxygen vacancies. Defects can be ionized, by electron or hole injection under the influence of X-ray or $\gamma$-radiation [8]. The absorption of X-rays generates a lot of new defects on the surface, free and bound electrons and holes, which may recombine to give near-band emission or transfer their energy to luminescence centres thereby inducing defect luminescence. The X-rays create stable defects, change the luminescence intensity, also spectral distribution. To inhibit electron-hole recombination the $\mathrm{Eu}^{3+}$ ion was used as an effective electron trap through importing new energetically favorable levels [9]. For all listed reasons, a comprehensive study on the fundamental photophysics and synthesis strategies of $\mathrm{Eu}^{3+}$ activated nanoparticles are essential.

Trivalent europium ions $\left(\mathrm{Eu}^{3+}\right)$ activated inorganic materials are one of the most important red emitting phosphors [10]. These phosphors exhibit abundant photochemical properties, as low toxicity, high photostability and sharp emission bands. The enthralling optical properties of $\mathrm{Eu}^{3+}$ ions derive from $\mathrm{f}-\mathrm{f}$ transitions between the $4 \mathrm{f}^{6}$ orbitals, which are theoretically parity forbidden and become partially

\footnotetext{
* Corresponding author. Institute of Solid State Physics, University of Latvia, 8 Kengaraga Street, Riga, LV-1063, Latvia.

E-mail address: tamarag@vin.bg.ac.rs (T. Gavrilović).
} 
allowed due to the small influence of the crystal field [11,12]. The emission lines of $\mathrm{Eu}^{3+}$ are very sharp which provide noticeable spectroscopic fingerprints for probing the local surrounding symmetry [13].

Also, the optical characteristics of luminescent materials strongly depend on the properties of the host, kind, concentration and electronic structure of incorporated ions [14,15]. Lanthanum orthophosphates $\left(\mathrm{LaPO}_{4}\right)$ doped with various trivalent lanthanide ions $\left(\mathrm{Ln}^{3+}\right)$ ions, serve as both an activator and sensitizer center and represents a significant class of luminescent nanomaterials, suitable for emission of photons in the UV, visible, and near-infrared (NIR) region. Up to now, $\mathrm{LaPO}_{4}: \mathrm{Eu}^{3+}$ nanoparticles have found applications as versatile luminescence labels for biomedical testing, in vitro and in vivo bioimaging [16], materials for lighting phosphors and optical amplification materials in telecommunication [17], nanoscale electronic and plasma display panels [18]. Detecting defect related luminescence has been used as a tool for the characterization of defects in different inorganic luminescent materials.

The aim of this work was to investigate the size effect and surface defects on the spectral distribution of emission of $\mathrm{LaPO}_{4}: \mathrm{Eu}^{3+}$ nanoparticles of different sizes by analyzing shapes and number of Stark's components in measured luminescent spectra. The high-resolution spectroscopy (measured at $10 \mathrm{~K}$ ) under excitations by ultraviolet $266 \mathrm{~nm}$-laser and X-rays, as well as thermo-stimulated luminescence technique, were used to study effects of surface defects on the luminescent properties and shapes of spectra.

\section{Experimental}

\subsection{Material and methods}

The $\mathrm{LaPO}_{4}: 10 \mathrm{~mol} \% \mathrm{Eu}^{3+}$ nanoparticles of different sizes and morphologies were synthesized by reverse micelle and co-precipitation technique by analogy to the methods presented in our previous paper [19].

The reverse micelles method: A typical synthesis performed at room temperature was as it follows: cyclohexane $(100 \mathrm{ml})$, Triton X-100 $(60 \mathrm{ml})$, and n-pentanol $(20 \mathrm{ml})$ and $0.1 \mathrm{M}$ aqueous solution of $\left(\mathrm{NH}_{4}\right)_{2} \mathrm{HPO}_{4}$ in a corresponding volume ratio (18:1) were mixed. In the next step, $0.15 \mathrm{M}$ aqueous solutions of $\mathrm{La}\left(\mathrm{NO}_{3}\right)_{3} \times 6 \mathrm{H}_{2} \mathrm{O}$ and $\mathrm{Eu}$ $\left(\mathrm{NO}_{3}\right)_{3} \times 6 \mathrm{H}_{2} \mathrm{O}$ (in corresponding concentration ratio, $10 \mathrm{~mol} \%$ of $\mathrm{Eu}^{3+}$ ions with respect to $\mathrm{La}^{3+}$ ions) were added into the obtained mixture under continuous magnetic stirring for $1 \mathrm{~h}$ at room temperature and after $24 \mathrm{~h}$ aging, the white colloid dispersion containing reverse micelles was obtained. The resulting precipitate was separated by centrifugation, washed by methanol and water and it was dried at $70{ }^{\circ} \mathrm{C}$ in the air for $24 \mathrm{~h}$. Throughout the manuscript, the sample prepared with the reverse micelle technique will be denoted as S1-M.

The co-precipitation method: An appropriate amount of $\left(\mathrm{NH}_{4}\right)_{2} \mathrm{HPO}_{4}$ was dissolved in water to obtain $0.05 \mathrm{M}$ solution $(\mathrm{pH}=12)$. A mixture of $0.05 \mathrm{M}$ aqueous solutions of $\mathrm{La}\left(\mathrm{NO}_{3}\right)_{3} \times 6 \mathrm{H}_{2} \mathrm{O}$ and $\mathrm{Eu}$ $\left(\mathrm{NO}_{3}\right)_{3} \times 6 \mathrm{H}_{2} \mathrm{O}$ (in concentration ratio of $10 \mathrm{~mol} \% \mathrm{Eu}^{3+}$ with respect to $\mathrm{La}^{3+}$ ions) was added drop-wise in a $\left(\mathrm{NH}_{4}\right)_{2} \mathrm{HPO}_{4}$ solution. A formed precipitate of $\mathrm{LaPO}_{4}: \mathrm{Eu}^{3+}$ was additionally heated and stirred at $70{ }^{\circ} \mathrm{C}$ for $1 \mathrm{~h}$ and it was separated from the suspension by centrifugation, and washed several times with distilled water to adjust the $\mathrm{pH}$ value to 7 . At the end, the collected powder of $\mathrm{LaPO}_{4}: \mathrm{Eu}^{3+}$ was dried at $70{ }^{\circ} \mathrm{C}$ for $20 \mathrm{~h}$ and additionally was annealed at $600{ }^{\circ} \mathrm{C}$ for $2 \mathrm{~h}$ and these two samples will be denoted as S2-ap and S3-600, respectively.

\subsection{Characterization methods and instrumentation}

Powder X-ray diffraction (XRD) measurements were performed on a Rigaku SmartLab diffractometer using $\mathrm{Cu}-\mathrm{K} \alpha 1,2$ radiation $(\lambda=0.15405 \mathrm{~nm})$. Diffraction data were recorded with a step size of $0.01^{\circ}$ and a counting time of $1 \mathrm{deg} / \mathrm{min}$ over the $2 \theta$ range of $10^{\circ}-90^{\circ}$. Transmission electron microscopy (TEM) studies were made on a Tecnai G20 (FEI) operated at an accelerating voltage of $200 \mathrm{kV}$. For these measurements, ethanol was used to transfer powder samples to the grids, and ultrasonic bath to separate agglomerates. An ultrathin carbon film on holey carbon grids (Agar S187-4) were used to promote measurements of extremely small-size nanoparticles.

Photoluminescent spectra were recorded using Andor Shamrock B303-I spectrometer utilizing a YAG:Nd laser LCS-DTL-382QT ( $266 \mathrm{~nm}$, $8 \mathrm{~ns}$ ) for photoluminescence excitation. The samples were cooled down using Janis closed cycle refrigerator CCS-100 operating within temperature range $\sim 9-325 \mathrm{~K}$. The Lake Shore 331 Temperature controller was used for temperature control as well as for sample heating (6 deg/ min) during thermally stimulated luminescence (TSL) measurements up to $320 \mathrm{~K}$. Luminescence spectra were recorded using Andor Shamrock B303-I spectrometer. The integration time was set to $1 \mathrm{~ms}$ for each spectrum recording. It is prominent that the filling of traps under UV excitation depends on both, the wavelength and temperature, indicating the energy necessary for the migration of charge carriers. Hence, for successful trap filling at low temperature, the samples were irradiated by X-rays (for $1 \mathrm{~h}$ ). The excitation source was X-ray tube with $\mathrm{W}$ target. The voltage of tube can be varied within $14 \mathrm{kV}-35 \mathrm{kV}$ and the current within 1-15 mA range, thus producing variable X-ray energy and intensity.

\section{Results and discussion}

\subsection{Microstructural and structural properties of $\mathrm{LaPO}_{4}: \mathrm{Eu}^{3+}$ particles}

Morphologies of synthesized $\mathrm{LaPO}_{4}: 10 \mathrm{~mol} \% \mathrm{Eu}^{3+}$ nanoparticles were studied by TEM and results are given in Fig. 1. Short nanorods of approximately $2 \mathrm{~nm} \times 15 \mathrm{~nm}$ and $4 \mathrm{~nm} \times 20 \mathrm{~nm}$ in size are obtained by reverse micelle and co-precipitation methods, respectively (Fig. 1a and b), while single spherical particles about $5 \mathrm{~nm}$ in size were obtained for
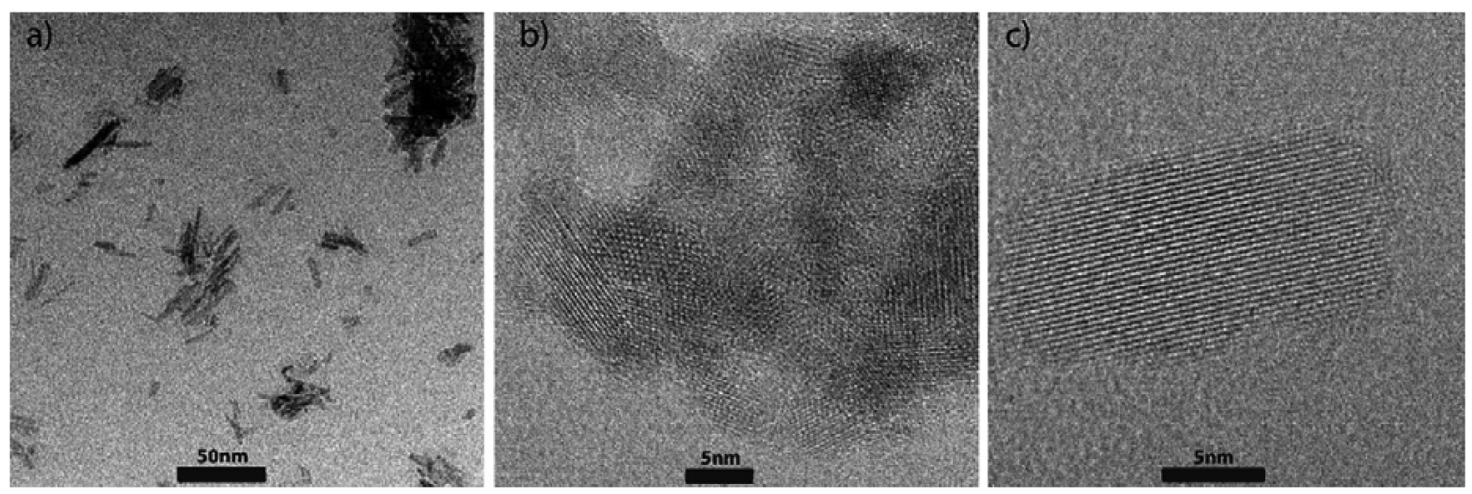

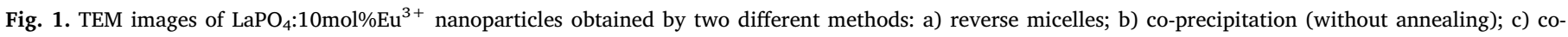
precipitation (with annealing at $\mathrm{T}=600^{\circ} \mathrm{C}$ ). 
Table 1

Sample names, method of synthesis, preparation temperature and obtained morphology and size of $\mathrm{LaPO}_{4}: 10 \mathrm{~mol}_{0} \mathrm{Eu}^{3+}$.

\begin{tabular}{|c|c|c|c|}
\hline $\begin{array}{l}\text { Samples } \\
\text { name }\end{array}$ & $\begin{array}{l}\text { Method of } \\
\text { synthesis }\end{array}$ & $\begin{array}{l}\text { Preparation } \\
\text { temperature, } \mathrm{T}\left({ }^{\circ} \mathrm{C}\right)\end{array}$ & $\begin{array}{l}\text { Morphology and } \\
\text { size }\end{array}$ \\
\hline S1-M & Reverse micelle & 70 & $\begin{array}{l}\text { Nanorods } \\
2 \mathrm{~nm} \times 15 \mathrm{~nm}\end{array}$ \\
\hline S2-ap & $\begin{array}{l}\text { Co-precipitation } \\
\text { without annealing }\end{array}$ & 70 & $\begin{array}{l}\text { Nanorods } \\
4 \mathrm{~nm} \times 20 \mathrm{~nm}\end{array}$ \\
\hline S3-600 & $\begin{array}{l}\text { Co-precipitation } \\
\text { with annealing }\end{array}$ & 600 & $\begin{array}{l}\text { Nanospheres } \\
5 \mathrm{~nm}\end{array}$ \\
\hline
\end{tabular}

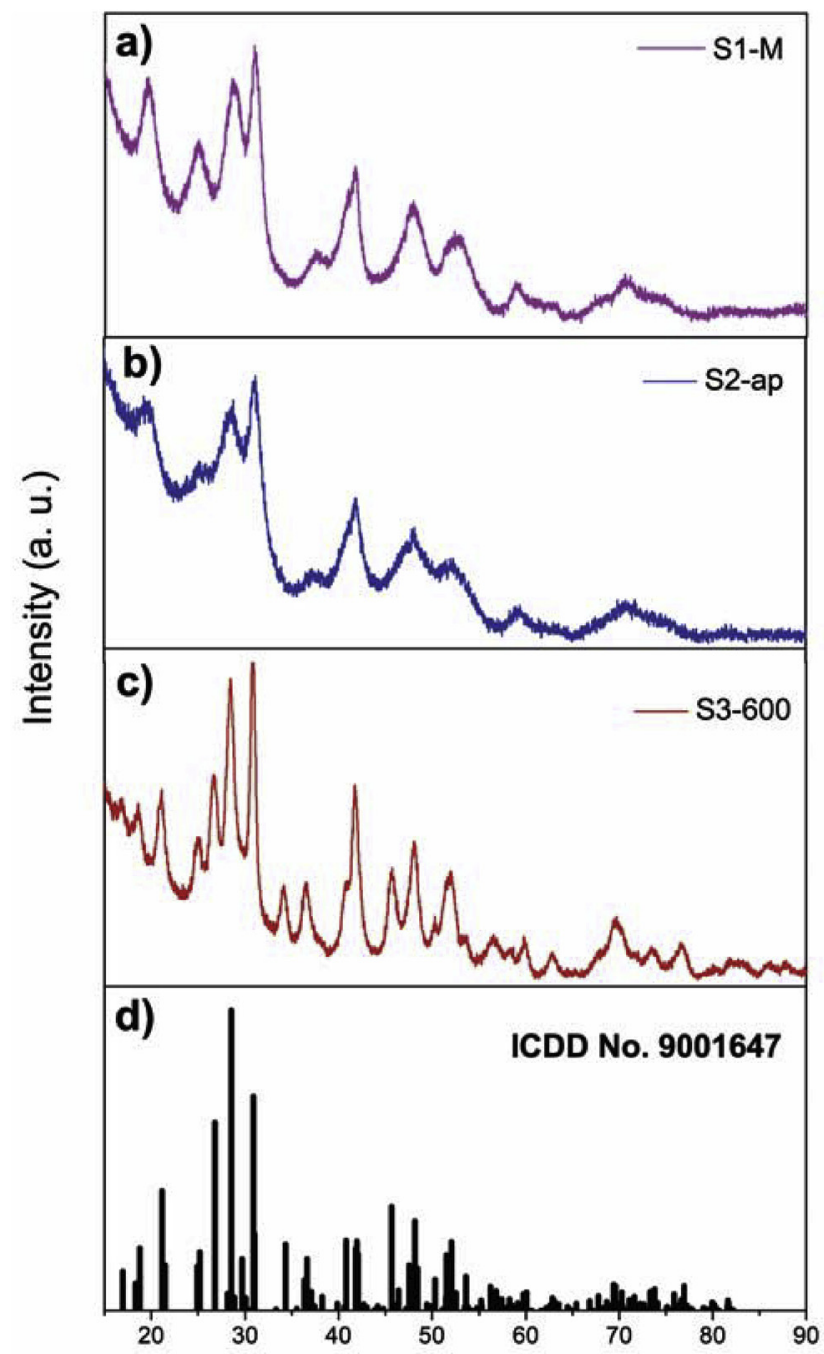

Fig. 2. X-ray diffraction patterns of $\mathrm{LaPO}_{4}: 10 \mathrm{~mol} \% \mathrm{Eu}^{3+}$ : a) nanorods $(2 \mathrm{~nm} \times 15 \mathrm{~nm})$, b) nanorods $(4 \mathrm{~nm} \times 20 \mathrm{~nm})$, c) nanospheres $(5 \mathrm{~nm})$ and d) vertical bars denote the standard data for monoclinic monazite structure of bulk $\mathrm{LaPO}_{4}$ (ICDD card No. 90001647).

sample annealed at $600{ }^{\circ} \mathrm{C}$ (Fig. 1c). Throughout the manuscript, the samples are denoted as in Table 1.

X-ray diffraction patterns of $\mathrm{LaPO}_{4}: 10 \mathrm{~mol} \% \mathrm{Eu}^{3+}$ samples having particles of different sizes are shown in Fig. 2. $\mathrm{LaPO}_{4}$ crystallizes in a pure monoclinic monazite phase of a space group P121/m1 (ICDD card No. 90001647). X-ray patterns are characterized with broad diffraction peaks indicating that samples are composed of small crystallite, and most likely containing an amorphous phase. With preparation temperature increase diffraction peaks became narrower and after $600{ }^{\circ} \mathrm{C}$ temperature heat treatment fully crystalline sample has been obtained.
Table 2

Structural parameters of $\mathrm{LaPO}_{4}: 10 \mathrm{~mol}_{\%} \mathrm{Eu}^{3+}$ nanoparticles obtained after Rietveld refinement of XRD data.

\begin{tabular}{llll}
\hline Parameters & $\begin{array}{l}\text { nanorods } \\
(2 \times 15 \mathrm{~nm})\end{array}$ & $\begin{array}{l}\text { nanorods } \\
(4 \times 20 \mathrm{~nm})\end{array}$ & $\begin{array}{l}\text { nanoparticles } \\
(5 \mathrm{~nm})\end{array}$ \\
\hline Crystallite size $(\mathrm{nm})$ & 2.5 & 2.7 & 5.2 \\
a (nm) & 0.6878 & 0.6956 & 0.6845 \\
b (nm) & 0.7070 & 0.7040 & 0.7075 \\
c (nm) & 0.6515 & 0.6498 & 0.6501 \\
\hline
\end{tabular}

The absence of impurity phases indicates that the dopant $\mathrm{Eu}^{3+}$ ions were successfully and uniformly incorporated into the $\mathrm{LaPO}_{4}$ matrix due to the equal valence $(+3)$ and similar ionic radii $(a)$ between $\mathrm{Eu}^{3+}(a=0.103 \mathrm{~nm})$ and $\mathrm{La}^{3+}$ ion $(a=0.115 \mathrm{~nm})$.

Structural parameters of all $\mathrm{LaPO}_{4}: 10 \mathrm{~mol} \% \mathrm{Eu}^{3+}$ samples were derived by the Rietveld refinement of XRD data, and results of the fitting are summarized in Table 2. Microstrain values range from 0.54 to $0.58 \%$ and suggest a good ion ordering in the nanocrystals. The average crystallite size, obtained from the Rietveld analysis, is in the $2.5-5.2 \mathrm{~nm}$ range, depending on the method of synthesis. The particle sizes assessed by TEM are similar as crystallite size obtained from XRD measurements for all nanoparticles, suggesting that each small particle comprises a single crystallite.

\subsection{Luminescent properties of $\mathrm{LaPO}_{4}: \mathrm{Eu}^{3+}$ nanoparticles under $266 \mathrm{~nm}$ - laser and $X$-rays excitations at $T=10 \mathrm{~K}$}

In this section, we will focus on the detail study of surface defects on luminescent properties of different sized $\mathrm{LaPO}_{4}: \mathrm{Eu}^{3+}$ nanoparticles. As it is already known, the structure of luminescence bands depends on local symmetry of incorporated ions. Additionally, the size of the crystallites in the samples is also influential because it determines the fraction of atoms, including dopants, at the surface which affect the luminescence properties of $\mathrm{Ln}^{3+}$ doped nanoparticles. Therefore, it is important to study the behavior of luminescent dopant ions to better understand the basic processes in nanomaterials, such as the defects influence on the distortions of $\mathrm{Eu}^{3+}$ ions in nanostructures.

The high-resolution spectroscopy (at $10 \mathrm{~K}$ ) under excitations by ultraviolet $266 \mathrm{~nm}$ laser and X-rays, as well as thermo-stimulated luminescence technique, were used to study effects of surface defects on the luminescent properties and shapes of spectra. Luminescent spectra for all samples were measured by 3 steps: 1) under laser excitation $(266 \mathrm{~nm}), 2)$ under X-ray-induced luminescence, 3) the samples were irradiated with X-rays in duration of $15 \mathrm{~min}$ and once again were measured under laser excitation $(266 \mathrm{~nm})$. Ultraviolet radiation was efficiently absorbed by a transition to the charge transfer state of the $\mathrm{Eu}^{3+}-\mathrm{O}^{2-}$.

For all synthesized samples, after non-radiative relaxation to the lower $4 \mathrm{f}$ levels, emission occurs from the $5 \mathrm{D}_{J}$ (mainly $5 \mathrm{D}_{0}$ ) to the ${ }^{7} \mathrm{~F}_{J}$ $(\mathrm{J}=0,1,2,3,4,5,6)$ states of $\mathrm{Eu}^{3+}$ ions. Luminescence spectral distributions of $\mathrm{Eu}^{3+}$-doped $\mathrm{LaPO}_{4}$ measured at $10 \mathrm{~K}$ are presented at Figs. 3-5 and the following emission transitions were detected: sensitive forbidden transition with very week intensity, ${ }^{5} \mathrm{D}_{0} \rightarrow{ }^{7} \mathrm{~F}_{0}$ at $578.4 \mathrm{~nm}$, magnetic-dipole transition ${ }^{5} \mathrm{D}_{0} \rightarrow{ }^{7} \mathrm{~F}_{1}$ at $(588-595 \mathrm{~nm})$, electric-dipole transition ${ }^{5} \mathrm{D}_{0} \rightarrow{ }^{7} \mathrm{~F}_{2}$ at $(611.5-620.5 \mathrm{~nm}),{ }^{5} \mathrm{D}_{0} \rightarrow{ }^{7} \mathrm{~F}_{3}$ at (648-652 nm) and ${ }^{5} \mathrm{D}_{0} \rightarrow{ }^{7} \mathrm{~F}_{4}$ at $(684-702.5 \mathrm{~nm})$.

The presence of the sensitive forbidden ${ }^{5} \mathrm{D}_{0} \rightarrow{ }^{7} \mathrm{~F}_{0}$ transition is not so common, but in our case, it is detected at $578.4 \mathrm{~nm}$. It is well-known that this transition is strictly forbidden according to the standard Judd-Ofelt theory and its existence is attributed to the phenomena of the selection rules breakdown [10]. The induced magnetic-dipole $\left({ }^{5} \mathrm{D}_{0} \rightarrow{ }^{7} \mathrm{~F}_{0}\right)$ transition with similar intensity and half width at half maximum at about $20 \mathrm{~cm}^{-1}$, is observed for all synthesized samples. The presence of this transition indicates that the $\mathrm{Eu}^{3+}$ ion occupies a site with $C_{n v}, C_{n}$ or $\mathrm{C}_{\mathrm{s}}$ symmetry [20]. In literature it was found that this 

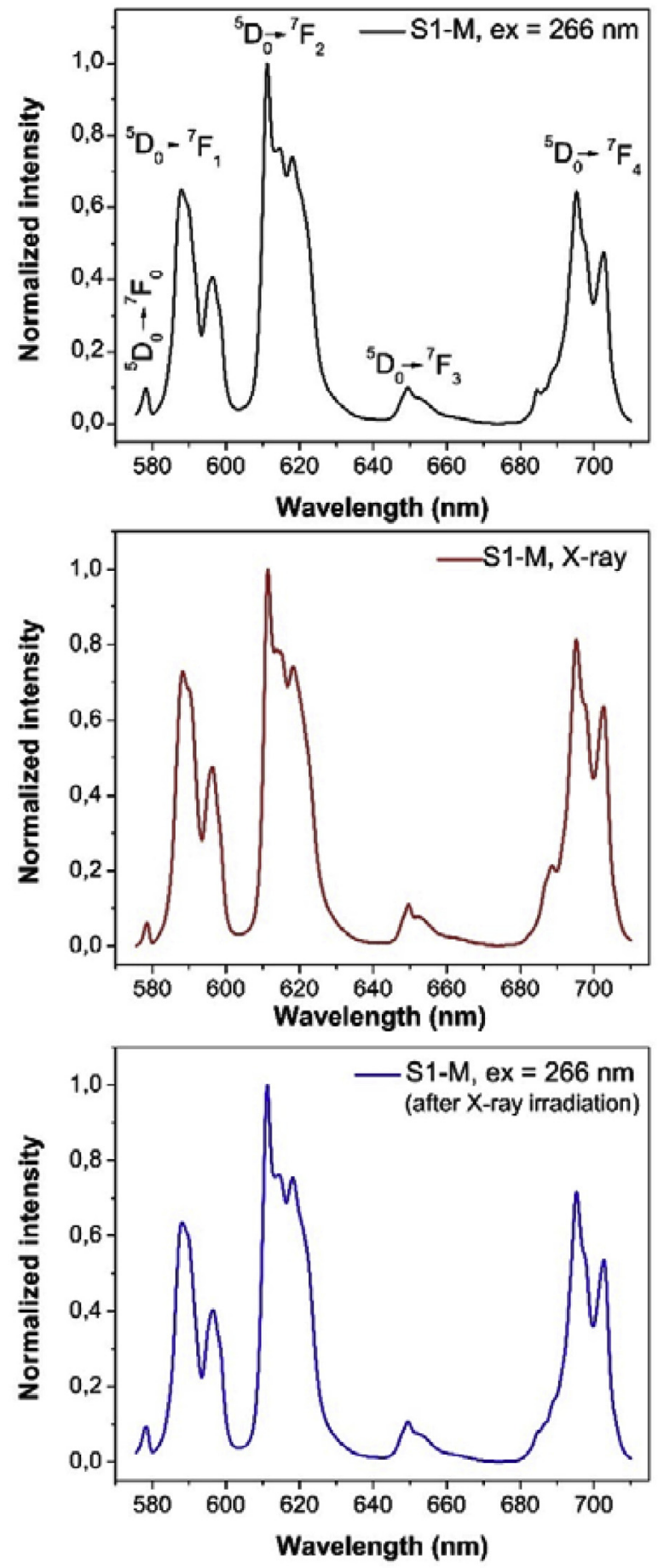

Fig. 3. Luminescence spectra of S1-M sample at $10 \mathrm{~K}$ under: a) laser excitation $(266 \mathrm{~nm}), \mathrm{b}) \mathrm{X}$-ray excitation, 3) irradiated with X-rays and measured under laser excitation $(266 \mathrm{~nm})$.

transition usually appears at lower temperatures than room temperature with line width from $0.12 \mathrm{~cm}^{-1}$ for $\mathrm{EuCl}_{3} \cdot 6 \mathrm{H}_{2} \mathrm{O}$ at $4.2 \mathrm{~K}$ up to $149 \mathrm{~cm}^{-1}$ in silicate and germanate glasses [21,22].

On Figs. 3-5 are given luminescent spectra at $\mathrm{T}=10 \mathrm{~K}$ for all three synthesized samples 1) under laser excitation $(266 \mathrm{~nm}), 2)$ under X-ray excitation, 3) the samples were irradiated with X-rays in duration of $15 \mathrm{~min}$ and once again photoluminescence intensities were measured under laser excitation ( $266 \mathrm{~nm}$ ). Fig. 3 displays luminescent spectra for S1-M sample under these three different excitations. There are no differences between those three spectra. Fig. 4 shows luminescent spectra
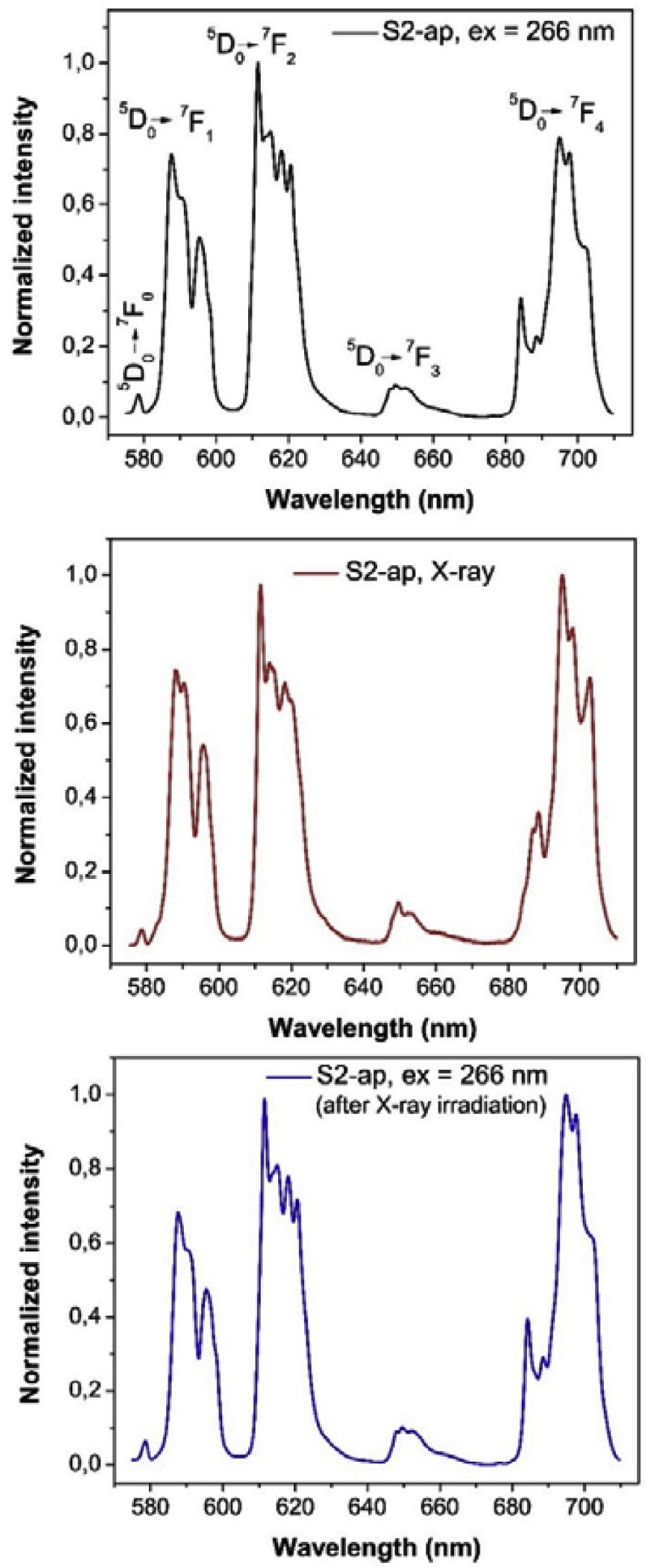

Fig. 4. Luminescence spectra of S2-ap sample at $\mathrm{T}=10 \mathrm{~K}$ under: a) laser excitation $(266 \mathrm{~nm})$, b) X-ray excitation, 3) irradiated with X-rays and measured under laser excitation $(266 \mathrm{~nm})$.

for S2-ap sample under three different excitations. The luminescence spectra under $266 \mathrm{~nm}$ and X-ray excitation are distinct indicating the differences between electron hole creation and Eu-O ligand excitation. The spectrum with different shapes of peaks for ${ }^{5} \mathrm{D}_{0} \rightarrow{ }^{7} \mathrm{~F}_{1}$ and ${ }^{5} \mathrm{D}_{0} \rightarrow{ }^{7} \mathrm{~F}_{4}$ transitions was observed under X-ray excitation. The absorption of Xrays leads to the generation of many new defect free and bound electrons and holes on the surface, which may recombine to give near-band emission or transfer their energy to luminescence centres thereby inducing defect luminescence. The X-rays created stable defects, changes the luminescence intensity and spectral distribution. Fig. 5 presents 

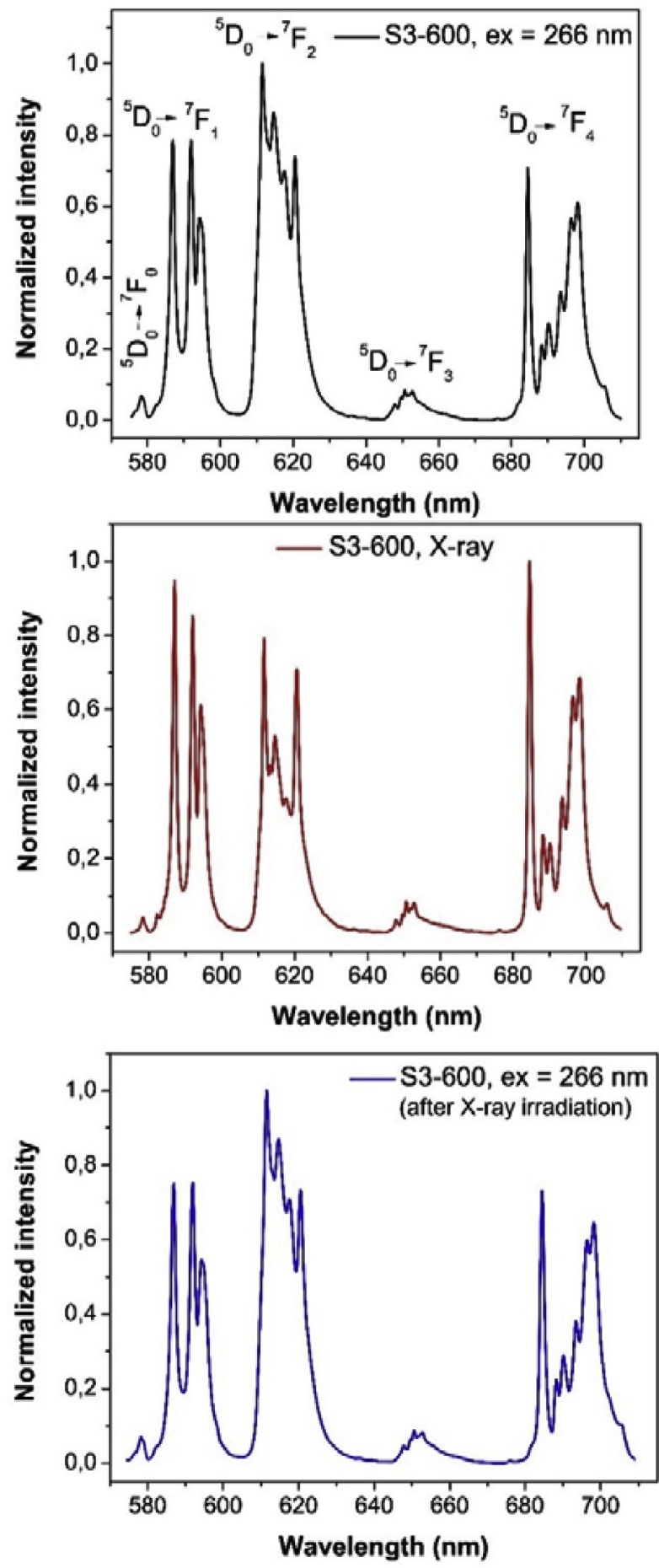

Fig. 5. Luminescence spectra of $\mathrm{S} 3-600$ sample at $10 \mathrm{~K}$ under: a) laser excitation $(266 \mathrm{~nm}), \mathrm{b}) \mathrm{X}$-ray excitation, 3) irradiated with X-rays and measured under laser excitation $(266 \mathrm{~nm})$.

luminescent spectra for S3-600 sample under three different excitation conditions. The most dominant transition in the X-ray-induced luminescent spectrum is ${ }^{5} \mathrm{D}_{0} \rightarrow{ }^{7} \mathrm{~F}_{1}$. Also, in this spectrum the difference in shapes and intensity of peak from ${ }^{5} \mathrm{D}_{0} \rightarrow{ }^{7} \mathrm{~F}_{1},{ }^{5} \mathrm{D}_{0} \rightarrow{ }^{7} \mathrm{~F}_{2}$ and ${ }^{5} \mathrm{D}_{0} \rightarrow{ }^{7} \mathrm{~F}_{4}$ were observed in comparison with spectra measured for the same sample under laser excitation at $266 \mathrm{~nm}$. This magnetic-dipole allowed ${ }^{5} \mathrm{D}_{0}-{ }^{7} \mathrm{~F}_{1}$ transition is the most prominent probably due to effects of phosphate groups in $\mathrm{LaPO}_{4}$ and formed defect as it was mentioned before. Also, the intensity of luminescent band for induced electric-dipole ${ }^{5} \mathrm{D}_{0} \rightarrow{ }^{7} \mathrm{~F}_{4}$ transition is very high and it is comparable with intensity of magnetic-dipole ${ }^{5} \mathrm{D}_{0} \rightarrow{ }^{7} \mathrm{~F}_{1}$ transition. The very high intensity of the ${ }^{5} \mathrm{D}_{0} \rightarrow{ }^{7} \mathrm{~F}_{4}$ transition was observed in $\mathrm{Ca}_{3} \mathrm{Sc}_{2} \mathrm{Si}_{3} \mathrm{O}_{12}: \mathrm{Eu}^{3+}$ sample due to a distortion of the cubic geometry of the $\mathrm{Eu}^{3+}$ site in this matrix [23]. The intensity of the ${ }^{5} \mathrm{D}_{0} \rightarrow{ }^{7} \mathrm{~F}_{4}$ transition could not be determined only by symmetry factors, but also by decreased energy loss in excited states and the chemical composition of the host matrix [10,24,25].

Due to the small difference between ionic sizes of $\mathrm{Eu}^{3+}$ ion $(0.103 \mathrm{~nm})$ and $\mathrm{La}^{3+}$ ion $(0.115 \mathrm{~nm})$, we consider that $\mathrm{Eu}^{3+}$ ions can occupy $\mathrm{La}^{3+}$ ion sites (C1 symmetry) which gives rise to a characteristic crystal splitting of the energy levels. It could be noted that the numbers of Stark's components of a ${ }^{7} \mathrm{~F}_{0}$ term is " 1 " for all symmetry classes and point groups and from this term could not be concluded anything about the change of lattice symmetry [24]. For synthesized nanorods of $2 \mathrm{~nm} \times 15 \mathrm{~nm}(\mathrm{~d} \times \mathrm{l})(\mathrm{S} 1-\mathrm{M}$ sample, Fig. 3) the smallest number of Stark's components of luminescence bands was observed. Numbers of Stark's components of a ${ }^{7} F_{J}$ term are $1,3,3,2$, 4, respectively, for $\mathrm{J}=0$, 1, 2, 3, 4. The ${ }^{5} D_{0} \rightarrow F_{1}$ luminescence band, which is convenient to follow up the change of lattice symmetry, is split into three Stark's components (the most evident for the X-ray-induced luminescent spectrum) which is typical for monoclinic symmetry class [10]. In comparation to S1-M sample, the splitting structure of luminescence bands in nanorods $(4 \times 20 \mathrm{~nm}(\mathrm{~d} \times 1)$, S2-ap sample, Fig. 4$)$ is the same for ${ }^{5} D_{0} \rightarrow{ }^{7} F_{1}$ luminescence band, but different for other terms. Numbers of Stark's components of a $7 \mathrm{~F}_{\mathrm{J}}$ terms are $1,3,4,3,5$, respectively, for $\mathrm{J}=0,1,2,3,4$ for S2-ap sample. The quantity and intensity of Stark's components for $5 \mathrm{~nm}$ nanospheres (S3-600 sample, Fig. 5) is greater than in the S2-ap sample and numbers of Stark's components of ${ }^{7} \mathrm{~F}_{\mathrm{J}}$ terms are $1,3,4,4,7$, respectively, for $J=0,1,2,3$, 4. In all three synthesized samples, it is easily observed that the splitting of luminescence band corresponding to ${ }^{5} \mathrm{D}_{0} \rightarrow^{7} \mathrm{~F}_{1}$ electronic transition is onto three Stark's components due to monoclinic symmetry of lattice.

From comparative overview of the luminescence spectra (Figs. 3-5) of all three synthesized $\mathrm{LaPO}_{4}: 10 \mathrm{~mol} \% \mathrm{Eu}$ samples of different sizes under $266 \mathrm{~nm}$ excitation at $10 \mathrm{~K}$ it can be concluded that: 1) the most dominant transition with strongest emission intensity is electric-dipole ${ }^{5} \mathrm{D}_{0} \rightarrow{ }^{7} \mathrm{~F}_{2}$ transition, 2) difference in shape, intensity and intensity ratio was obtained, 3) the most resolved splitting was observed in the sample S3-600. Further, the luminescent intensity for S3-600 sample is the highest (in comparation with another samples before normalizing the spectra), probably because of the quenching caused by presence of quenching centers, impurities from precursors such as $\mathrm{NO}^{3-}$ and $\mathrm{OH}^{-}$ ions, which are effectively reduced at high temperature $\left(\mathrm{T}=600^{\circ} \mathrm{C}\right.$ was used for preparing this sample).

As the optical properties are strongly dependent on particle size, a particle size distribution is expected to cause inhomogeneous broadening of emission peaks. The emission spectra often exhibit well defined peaks associated with band-edge luminescence and defects recombination. However, samples with appropriate mixture of bound and free defects (electrons, holes and vacancies) lead to broadening of emission spectra due to merging of peaks. Also, these peaks are broadened homogeneously due to particle size distribution; however, detail quantitative spectral analysis will be our next aim [26].

\subsection{Kinetics and Judd-Ofelt analysis}

Judd-Ofelt (JO) parameters provide information about local environment of the $\mathrm{Eu}^{3+}$ ions and can be further used to derive other useful parameters such as spontaneous emission probability and the emission quantum efficiency of the lanthanide ion.

JO parameters were calculated by the following formula [27]:

$\Omega_{\lambda}=\frac{D_{m d} \nu_{1}^{3}}{e^{2} \nu_{\lambda}^{3}} \frac{9 n^{3}}{n\left(n^{2}+2\right)^{2}\left|\left\langle\Psi J\left\|U^{(\lambda)}\right\| \Psi^{\prime} J^{\prime}\right\rangle\right|^{2}} \frac{\int I_{\lambda}(\nu) d \nu}{\int I_{1}(\nu) d \nu}$

Where $D_{m d}$ is the magnetic dipole strength $9.6 \times 10^{-42} \mathrm{esu}^{2} \mathrm{~cm}^{2}$, e is elementary charge $4.803 \times 10^{-10} \mathrm{esu},\left|\Psi J U^{(\lambda)} \Psi^{\prime} J^{\prime}\right|^{2}$ is the squared 
Table 3

Kinetic parameters of $\mathrm{LaPO}_{4}$ samples of different sizes irradiated with X-rays, obtained by using the glow curve shape method (given by Nagabhushana et al.) [35].

\begin{tabular}{lllllllll}
\hline Sample & TSL glow curve & $\operatorname{Tm}(\mathrm{K})$ & $\mu_{\mathrm{g}}$ & \multicolumn{5}{c}{ Activation energy, $E_{\alpha}(\mathrm{eV})$} \\
\cline { 5 - 8 } & & & & & $\mathrm{E}_{\tau}$ & $\mathrm{E}_{\delta}$ & $\mathrm{E}_{\omega}$ & $\mathrm{E}_{\text {ave }}$ \\
\hline 1-M & 1 & 212.17 & 0.428 & 0.444 & 0.454 & 0.451 & 0.451 \\
S2-ap & 1 & 212.11 & 0.488 & 0.396 & 0.419 & 4090 & 0.408 \\
S3-600 & 1 & 58.72 & 0.663 & 0.071 & 0.028 & 0.024 & 0.041 \\
& 2 & 172.28 & 0.576 & 0.165 & 0.182 & 0.175 & 0.174 \\
\hline
\end{tabular}

reduced matrix element for $\mathrm{Eu}^{3+}$ independent of host and $\mathrm{n}=1.85$ is the refractive index of $\mathrm{LaPO}_{4}$ [28].

To determine the electric dipole strength, the following equation was used:

$D_{e d}=e^{2} \sum_{\lambda=2,4,6} \Omega_{\lambda}\left|\left\langle\Psi J \| U^{(\lambda)}|| J^{\prime}\right\rangle\right|^{2}$

The spontaneous emission probability can then be calculated as:

$A\left(\Psi J, \Psi^{\prime} J^{\prime}\right)=\frac{64 \pi^{4} \nu^{3}}{3 h(2 J+1)}\left[\frac{n\left(n^{2}+2\right)^{2}}{9} D_{e d}+n^{3} D_{m d}\right]$

Where $v$ is the average transition energy, $h$ is the Planck constant $\left(6.63 \times 10^{-27}\right.$ erg s) and $2 \mathrm{~J}+1$ is the degeneracy of the initial state ${ }^{5} \mathrm{D}_{0}$. The total radiative rate $A_{R}$ can be further used to determine nonradiative rate and the emission quantum efficiency (see Table 4).

The radiative lifetimes were calculated by fitting a double exponential to the measured decay curves of the samples and then averaged using the following equation:

$\tau_{\text {avg }}=\frac{A_{1} \tau_{1}^{2}+A_{2} \tau_{2}^{2}}{A_{1} \tau_{1}+A_{2} \tau_{2}}$

Nonradiative rate $A_{N R}$ and the resulting quantum efficiency was calculated as:

$A_{N R}=\frac{1}{\tau}-A_{R}$

$\eta=\frac{A_{R}}{\left(A_{R}+A_{N R}\right)}$

It has been indicated that $\Omega_{2}$ parameter is related to symmetry and the hypersensitive transitions while $\Omega_{4}$ is thought to capture long-range effects and electronic density surrounding the rare-earth site $[29,30]$.

$\Omega_{2}<\Omega_{4}$ indicates that $\mathrm{Eu}^{3+}$ ions are in a symmetric environment, which is thought to be plausible due to the ions occupying $\mathrm{La}^{3+}$ sites and therefore not causing any charge imbalances [30]. The increase in $\Omega_{2}$ also indicates an increase in covalency which is the highest for sample S3-600.

With the increase of $\Omega_{2} \mathrm{Eu}^{3+}$ surroundings are assumed to become more asymmetric and therefore resulting in an increase in intensity of the electric-dipole transition ${ }^{5} \mathrm{D}_{0} \rightarrow{ }^{7} \mathrm{~F}_{2}[29,31]$.

\subsection{Thermally stimulated emission}

Additionally, the thermo-stimulated luminescence on all three

Table 4

$\Omega_{2}, \Omega_{4}$ JO parameters, experimental decay lifetimes $(\tau)$ for ${ }^{5} \mathrm{D}_{0} \rightarrow{ }^{7} \mathrm{~F}_{2}$, radiative transition rates $\left(A_{R}\right)$, nonradiative rates $\left(A_{N R}\right)$, emission quantum efficiency $(\eta)$.

\begin{tabular}{lllllll}
\hline & $\begin{array}{l}\Omega_{2}\left(\times 10^{-20}\right. \\
\left.\mathrm{cm}^{2}\right)\end{array}$ & $\begin{array}{l}\Omega_{4}\left(\times 10^{-20}\right. \\
\left.\mathrm{cm}^{2}\right)\end{array}$ & $\tau, \mathrm{ms}$ & $\mathrm{A}_{\mathrm{R}}\left(\mathrm{s}^{-1}\right)$ & $\mathrm{A}_{\mathrm{NR}}\left(\mathrm{s}^{-1}\right)$ & $\eta, \%$ \\
\hline S1-M & 2.65 & 2.52 & 2.1 & 129 & 363 & 26.2 \\
S2-ap & 2.25 & 3.18 & 2.0 & 128 & 341 & 27.3 \\
S3-600 & 2.72 & 2.85 & 3.7 & 132 & 138 & 48.8 \\
\hline
\end{tabular}

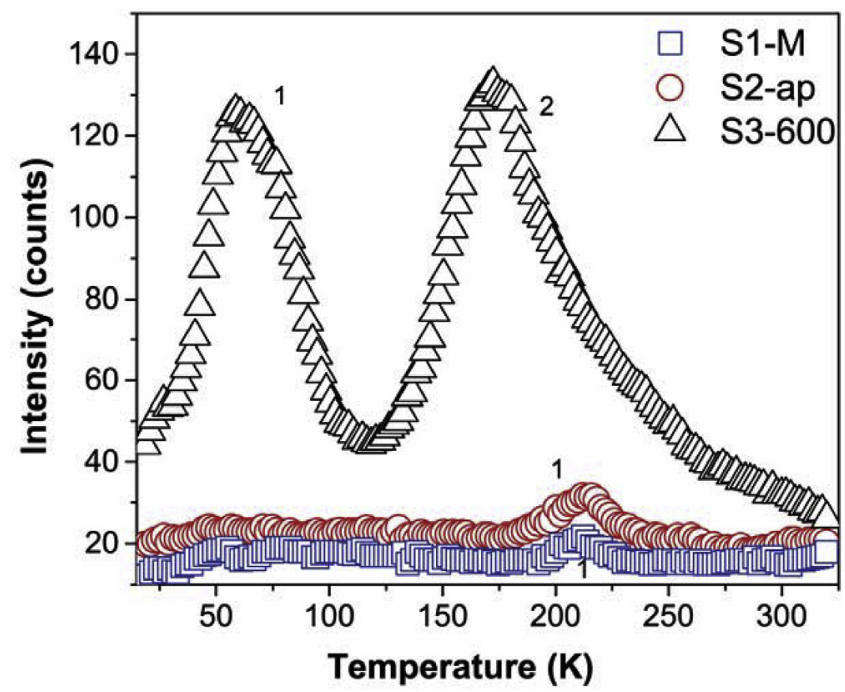

Fig. 6. X-ray induced TLS spectra of $\mathrm{LaPO}_{4}: \mathrm{Eu}^{3+}$ samples with different sizes.

synthesized samples was observed.

Thermo-stimulated luminescence (TSL) process is occurring when the thermally excited emission of lights follows the previous absorption of energy from radiation. Generally, experiments of TLS can give useful information on the different type of defects present within crystal structure, such as: electronic levels, trap depth of electrons and holes, recombination centres and kinetics their formation. In most materials, the appearance of defects or impurities is considered essential for thermoluminescence to occur [32]. In a thermo-stimulated process, the energy reserved in the crystal is liberated with the emission of light throughout heating of the irradiated material and the intensity of the emitted light represented as a function of temperature forms a glow curve.

The TLS signal was recorded for most dominant peak at $611.5 \mathrm{~nm}$ for all synthetized samples. From Fig. 6 it is clearly observed that the $\mathrm{Eu}^{3+}$ doping creates shallow traps for S1-M and S2-ap samples and two deep traps for S3-600 sample which are located at lower temperatures. The strongest thermo-stimulated luminescence signal was obtained for S3-600 sample, shown on Fig. 6. Two prominent and well resolved TSL glows with peaks at $58.7 \mathrm{~K}$ and $172.3 \mathrm{~K}$ were observed in X-rays irradiated S3-600 sample, while small TSL glows with peak at 212.1 K and 212.2 K were observed in X-rays irradiated S1-M and S2-ap samples, respectively. These glows could be attributed to free, as well as bound electrons and holes or to the recombination of electrons of ionized oxygen vacancies with photogenerated holes [33]. It could be the same case for all samples, because there is broadening of emission spectra in all samples which could be assigned to defects, but to obtain specific information about type of defects additional investigations are necessary. High surface to volume ratio of this small nanoparticles play a major role in the concentration of ionized oxygen vacancies [33,34].

For the description of TSL characteristics, for our samples with different nanoparticle size, we calculated the values of kinetic parameters known as trapping parameters. Activation energy of the traps involved in TSL emission, temperature corresponding to glow peak maximum (Tm) and symmetry factor $\left(\mu_{\mathrm{g}}\right)$ are summarized in Table 3 . To obtain these parameters, the glow curve shape method was used [35].

TSL intensity $\mathrm{T}_{1}$ and $\mathrm{T}_{2}$ at the low and high temperature side of the glow peak maximum (Tm) were estimated. Obtained values are used to determine the widths using following equations: $\omega=T_{1}-T_{2}, \delta=T_{2}$ $T_{m}$ and $\tau=T_{m}-T_{1}$ as well as the symmetry factor of the glow-peak $\mu_{g}=$ $\delta / \omega$. Then, the activation energy $\mathrm{E}_{\mathrm{a}}$ was calculated by peak shape equations using obtained shape parameters $\tau, \delta$, and $\omega$ [35]. The peak shape equations can be summarized as: 
$E_{\alpha}=c_{\alpha}+\left(\frac{k T_{m}^{2}}{\alpha}\right)-b_{\alpha}\left(2 k T_{m}\right)$

Where $k$ is the Boltzmann constant $\left(8.6 \times 10^{-5} \mathrm{eV} \mathrm{K}^{-1}\right)$, " $\alpha$ " could be $\tau$, $\delta$ or $\omega$ and $c_{\alpha}$ will be given as:

$$
\begin{aligned}
& \mathrm{c}_{\tau}=1.51+3.0\left(\mu_{\mathrm{g}}-0.42\right), \mathrm{b}_{\tau}=1.58+4.2\left(\mu_{\mathrm{g}}-0.42\right) \\
& \mathrm{c}_{\delta}=0.976+7.3\left(\mu_{\mathrm{g}}-0.42\right), \mathrm{b}_{\delta}=0 \\
& \mathrm{c}_{\omega}=2.52+10.2\left(\mu_{\mathrm{g}}-0.42\right), \mathrm{b}_{\omega}=1
\end{aligned}
$$

The values of $E_{\alpha}$ for $\mathrm{LaPO}_{4}: \mathrm{Eu}^{3+}$ samples, obtained by three different synthetic routes, are given in Table 3 . From the calculated values, it is obvious that a considerable amount of re-trapping takes place in all samples. It is believed that there are some deep and shallow traps and for this reason there could be re-trapping of the electrons at deep traps (for S3-600 sample) going to upper shallow traps by stimulation due to thermal energy. The traps could be electron traps or hole traps or of both kinds [36]. However, it should be mentioned that at this point it is required to perform more detailed investigations using other methods to understand TLS mechanism and obtain some useful insights to assist in the interpretation of experimentally observed thermo-stimulated light emission of $\mathrm{LaPO}_{4}: \mathrm{Eu}^{3+}$.

\section{Conclusions}

$\mathrm{LaPO}_{4}: \mathrm{Eu}^{3+}$ samples with particles of different morphology and size were prepared by reverse micelle and co-precipitation synthetic methods: spherical nanoparticles of $5 \mathrm{~nm}$ in size and nanorods $2 \mathrm{~nm} \times 15 \mathrm{~nm}$ and $4 \mathrm{~nm} \times 20 \mathrm{~nm}$. XRD measurements evidenced that all $\mathrm{LaPO}_{4}$ particles crystallized in the pure monoclinic monazite phase suggesting that the dopant $\mathrm{Eu}^{3+}$ ions are successfully incorporated into the host lattice, due to equal valence and similar ionic radii between $\mathrm{Eu}^{3+}$ and $\mathrm{La}^{3+}$ ions.

The size and surface defects effects on the intensity of luminescence and shapes and structure of spectra, and number of Stark's components, were analyzed by high resolution spectroscopy under laser $(266 \mathrm{~nm})$ and X-rays excitation. It was easily observed in all three synthesized samples that the splitting of luminescence band corresponding to electronic transition ${ }^{5} \mathrm{D}_{0} \rightarrow{ }^{7} \mathrm{~F}_{1}$ is onto three Stark's components due to monoclinic symmetry of lattice. The quantity of Stark's components is the largest in the $5 \mathrm{~nm}$ nanoparticles. Also, in this sample, the luminescent intensity is the highest probably because of effectively reduced quenching caused by the presence of quenching centers. The thermostimulated luminescence for all synthesized samples was studied for the most dominant peak at $611.5 \mathrm{~nm}$. It was revealed that $\mathrm{Eu}^{3+}$ doping creates traps in all samples. The TSL glow peak intensity is found to be enhanced in the case of heat-treated sample when compared with asprepared samples. The glow curves were analyzed and trap parameters were estimated and discussed throughout the paper. The glows could be attributed to free and bound electrons and holes or to the recombination of electrons of ionized oxygen vacancies with photogenerated holes. However, it should be mentioned that at this point it is required to perform more detailed investigations to understand TLS mechanism and obtain useful insights to assist in the interpretation of experimentally observed thermo-stimulated light emission of $\mathrm{LaPO}_{4}: \mathrm{Eu}^{3+}$. The properties and functions of materials often result from various forms of structural defects. Nonradiative rate $A_{N R}$ and the resulting quantum efficiency was calculated using Judd-Ofelt analysis. The highest values of decay lifetimes $(\tau)$ and emission quantum efficiency for ${ }^{5} \mathrm{D}_{0} \rightarrow{ }^{7} \mathrm{~F}_{2}$ were obtained for heat-treated sample. The elucidation of luminescence intensity dependence on the nanoparticle size is not only fundamental, but also an applied task important for estimation of possible application of nanoparticles for creation of new nanocomposite luminescence materials or even the nanoscintillators for medical use.

\section{Acknowledgments}

T. G. acknowledges the ERDF PostDoc project No. 1.1.1.2/VIAA/1/ 16/215 (1.1.1.2/16/I/001). K. S. and K. L. acknowledge the Latvian National Research Program IMIS2. The authors from Vinča Institute of Nuclear Sciences acknowledge the financial support of the Ministry of Education, Science and Technological Development of the Republic of Serbia (Project No: 45020 and 172056).

\section{References}

[1] X.F. Duan, Y. Huang, Y. Cui, J.F. Wang, C.M. Lieber, Indium phosphide nanowires as building blocks for nanoscale electronic and optoelectronic devices, Nature 409 (2001) 66-69.

[2] H. Kind, H. Yan, B. Messer, M. Law, P. Yang, Nanowire ultraviolet photodetectors and optical switches, Adv. Mater. 14 (2002) 158-160.

[3] X.Y. Kong, Y. Ding, R. Yang, Z.L. Wang, Single-crystal nanorings formed by epitaxial self-coiling of polar nanobelts, Science 303 (2004) 1348-1351.

[4] M.Y. Guan, J.H. Sun, F.F. Tao, Z. Xu, A host crystal for the rare-earth ion dopants: synthesis of pure and $\mathrm{Ln}$-doped urchinlike $\mathrm{BiPO}_{4}$ structure and its photoluminescence, Cryst. Growth Des. 8 (2008) 2694-2697.

[5] M. Schäfer-Korting (Ed.), Drug Delivery in Handbook of Experimental Pharmacology, Springer-Verlag Berlin Heidelberg, Germany, 2010.

[6] A.B. Djurišić, K.H.Tam Y.H Leung, Y.F. Hsu, L. Ding, W.K. Ge, Y.C. Zhong, K.S. Wong, W.K. Chan, H.L. Tam, Defect emissions in ZnO nanostructures, Nanotechnology 18 (2007) 095702 (8pp).

[7] A. Leach, X. Shen, A. Faust, M.C. Cleveland, A.D. La Croix, U. Banin, S.T. Pantelides, J.E. Macdonald, Defect luminescence from wurtzite $\mathrm{CuInS}_{2}$ nanocrystals: combined experimental and theoretical analysis, J. Phys. Chem. C 120 (2016) 5207-5212.

[8] P. Procházka, D. Mareček, Z. Lišková, J. Čechal, T. Šikola, X-ray induced electrostatic graphene doping via defect charging in gate dielectric, Sci. Rep. 7 (2017) 563.

[9] L.V. Trandafilović, D.J. Jovanović, X. Zhang, S. Ptasińska, M.D. Dramićanin, Enhanced photocatalytic degradation of methylene blue and methyl orange by ZnO: Eu nanoparticles, Appl. Catal. B Environ. 203 (2017) 740-752.

[10] K. Binnemans, Interpretation of europium(III) spectra, Coord. Chem. Rev. 295 (2015) 1-45.

[11] P.A. Tanner, Some misconceptions concerning the electronic spectra of tri-positive europium and cerium, Chem. Soc. Rev. 42 (2013) 5090-5101.

[12] S.Y. Han, R.R. Deng, X.J. Xie, X.G. Liu, Enhancing luminescence in lanthanidedoped upconversion nanoparticles, Angew. Chem. Int. Ed. 53 (2014) 11702-11715.

[13] G. Vicentini, L.B. Zinner, J.Z. Schpector, K. Zinner, Luminescence and structure of europium compounds, Coord. Chem. Rev. 196 (2000) 353-382.

[14] X. Chen, Z. Xia, M. Yi, X. Wu, H. Xin, Rare-earth free self-activated and rare-earth activated $\mathrm{Ca}_{2} \mathrm{NaZn}_{2} \mathrm{~V}_{3} \mathrm{O}_{12}$ vanadate phosphors and their color-tunable luminescence properties, J. Phys. Chem. Solid. 74 (2013) 1439-1443.

[15] T.V. Gavrilović, D.J. Jovanović, V. Lojpur, A. Nikolić, M.D. Dramićanin, Influence of $\mathrm{Er}^{3+} / \mathrm{Yb}^{3+}$ concentration ratio on the downconversion and up-conversion luminescence and lifetime in $\mathrm{GdVO}_{4}: \mathrm{Er}^{3+} / \mathrm{Yb}^{3+}$ microcrystals, Sci. Sinter. 47 (2015) 221-228.

[16] D. Tu, W. Zheng, P. Huang, X. Chen, Europium-activated luminescent nanoprobes: from fundamentals to bioapplications, Coord. Chem. Rev. (2017) (in press), https:// doi.org/10.1016/j.ccr.2017.10.027 avaliable online from 20 November 2017.

[17] M. Ferhi, K.H. Naifer, M. Férid, Combustion synthesis and luminescence properties of $\mathrm{LaPO}_{4}$ : Eu (5\%), J. Rare Earths 27 (2009) 182-186.

[18] S.E. Létant, T.W. van Buuren, L.J. Terminello, Nanochannel arrays on silicon platforms by electrochemistry, Nano Lett. 4 (2004) 1705-1707.

[19] T. Gavrilović, J. Periša, J. Papan, K. Vuković, K. Smits, D.J. Jovanović, M.D. Dramićanin, Particle size effects on the structure and emission of $\mathrm{Eu}^{3+}: \mathrm{LaPO}_{4}$ and $\mathrm{EuPO}_{4}$ phosphors, J. Lumin. 195 (2018) 420-429.

[20] K. Binnemans, C.G. Walrand, Applications of the $\mathrm{Eu}^{3+}$ ion for site symmetry determination, J. Rare Earths 14 (1996) 173-180.

[21] K.H. Hellwege, P. Hill, S. Huefner, Temperature dependence of the wavenumber and linewidth of the ${ }^{7} \mathrm{~F}_{\mathrm{o}} \rightarrow{ }^{5} \mathrm{Do}$ transition in $\mathrm{Eu}^{3+}$, Solid State Commun. 5 (1967) 687-689.

[22] R. Reisfeld, R.A. Velapoldi, L. Boehm, M. Ish-Shalom, Transition probabilities of europium in phosphate glasses, J. Phys. Chem. 75 (1971) 3980-3983.

[23] M. Bettinelli, A. Speghini, F. Piccinelli, A.N.C. Neto, O.L. Malta, Luminescence spectroscopy of $\mathrm{Eu}^{3+}$ in $\mathrm{Ca}_{3} \mathrm{Sc}_{2} \mathrm{Si}_{3} \mathrm{O}_{12}$, J. Lumin. 131 (2011) 1026-1028.

[24] K. Riwotzki, H. Meyssamy, A. Kornowski, M. Haase, Liquid-phase synthesis of doped nanoparticles: colloids of luminescing $\mathrm{LaPO}_{4}: \mathrm{Eu}$ and $\mathrm{CePO}_{4}: \mathrm{Tb}$ particles with a narrow particle size distribution, J. Phys. Chem. B 104 (2000) 2824-2828.

[25] H. Meyssamy, K. Riwotzki, A. Kornowski, S. Naused, Markus Haase, Wet-chemical synthesis of doped colloidal nanomaterials: particles and fibers of $\mathrm{LaPO}_{4}: \mathrm{Eu}$, $\mathrm{LaPO}_{4}: \mathrm{Ce}$, and $\mathrm{LaPO}_{4}: \mathrm{Ce}, \mathrm{Tb}$, Adv. Materials 11 (1999) 840.

[26] S.J. Musevi, A. Aslani, H. Motahari, H. Salimi, Offer a novel method for size appraise of NiO nanoparticles by PL analysis: synthesis by sonochemical method, J. Saudi Chem. Soc. 20 (2016) 245-252.

[27] Lj Đačanin, S.R. Lukić, D.M. Petrović, M. Nikolić, M.D. Dramićanin, Judd-Ofelt analysis of luminescence emission from $\mathrm{Zn}_{2} \mathrm{SiO}_{4}: \mathrm{Eu}^{3+}$ nanoparticles obtained by a polymer-assisted sol-gel method, Physica B 406 (2011) 2319-2322.

[28] N. Saltmarsh, G.A. Kumar, M. Kailasnath, Vittal Shenoy, C. Santhosh, D.K. Sardar, Spectroscopic characterizations of Er doped $\mathrm{LaPO}_{4}$ submicron phosphors prepared 
by homogeneous precipitation method, Opt. Mater. 53 (2016) 24-29.

[29] S. Tanabe, T. Ohyagi, N. Soga, T. Hanada, Compositional dependence of Judd-Ofelt parameters of Er + ions in alkali-metal borate glasses, Phys. Rev. B 46 (1992) 3305.

[30] S.K. Gupta, P.S. Ghosh, M. Sahu, K. Bhattacharyya, R. Tewarib, V. Natarajan, Intense red emitting monoclinic $\mathrm{LaPO}_{4}: \mathrm{Eu}^{3+}$ nanoparticles: host-dopant energy transfer dynamics and photoluminescence properties, RSC Adv. 5 (2015) $58832-58842$

[31] S.G. Prasanna Kumar, R.H. Krishna, N. Kottam, P.K. Murthy, C. Manjunatha, R. Preetham, C. Shivakumara, T. Thomas, Understanding the photoluminescence behaviour in nano $\mathrm{CaZrO}_{3}: \mathrm{Eu}^{3+}$ pigments by Judd-Ofelt intensity parameters, Dyes Pigments 150 (2018) 306-314.

[32] J.K. Rike, F. Daniels, Thermoluminescence studies of aluminum oxide, J. Phys.
Chem. 61 (1957) 629-633.

[33] I.E. Kolesnikov, A.V. Povolotskiy, D.V. Mamonova, E. Lähderanta, A.A. Manshina, M.D. Mikhailov, Photoluminescence properties of $\mathrm{Eu}^{3+}$ ions in yttrium oxide nanoparticles: defect vs. normal sites, RSC Adv. 6 (2016) 76533-76541.

[34] A. Phuruangrat, N. Ekthammathat, S. Thongtem, T. Thongtem, Preparation of $\mathrm{LaPO}_{4}$ nanowires with high aspect ratio by a facile hydrothermal method and their photoluminescence, Res. Chem. Intermed. 39 (2013) 1363-1371.

[35] K.R. Nagabhushana, B.N. Lakshminarasappa, F. Singh, Thermoluminescence studies in swift heavy ion irradiated aluminum oxide, Radiat. Meas. 43 (2008) S651-S655.

[36] N. Salah, P.D. Sahare, S. Nawaz, S.P. Lochab, Luminescence characteristics of $\mathrm{K}_{2} \mathrm{Ca}_{2}\left(\mathrm{SO}_{4}\right)_{3}$ : Eu, Tb micro- and nanocrystalline phosphor, Radiat. Eff. Defect Solid 159 (2004) 321-334. 